\title{
A 22-channel receive array with Helmholtz transmit coil for anesthetized macaque MRI
} at $3 \mathrm{~T}$

\author{
Thomas Janssens ${ }^{a, b}$, Boris Keil ${ }^{a, c}$, Peter Serano ${ }^{a}$, Azma Mareyam $^{a}$, \\ Jennifer A. McNab ${ }^{a, c, d}$, Lawrence L. Wald ${ }^{a, c, e t}$ and Wim Vanduffel ${ }^{a, b, c_{*} \dagger}$
}

\begin{abstract}
The macaque monkey is an important model for cognitive and sensory neuroscience that has been used extensively in behavioral, electrophysiological, molecular and, more recently, neuroimaging studies. However, macaque MRI has unique technical differences relative to human MRI, such as the geometry of highly parallel receive arrays, which must be addressed to optimize imaging performance. A 22-channel receive coil array was constructed specifically for rapid high-resolution anesthetized macaque monkey MRI at $3 \mathrm{~T}$. A local Helmholtz transmit coil was used for excitation. Signal-to-noise ratios (SNRs) and noise amplification for parallel imaging were compared with those of single- and four-channel receive coils routinely used for macaque MRI. The 22-channel coil yielded significant improvements in SNR throughout the brain. Using this coil, the SNR in peripheral brain was 2.4 and 1.7 times greater than that obtained with single- or four-channel coils, respectively. In the central brain, the SNR gain was 1.5 times that of both the single- and four-channel coils. Finally, the performance of the array for functional, anatomical and diffusion-weighted imaging was evaluated. For all three modalities, the use of the 22-channel array allowed for high-resolution and accelerated image acquisition. Copyright $\odot 2013$ John Wiley \& Sons, Ltd.
\end{abstract}

Keywords: RF receive coils; array coils; animal model study; macaque

\section{INTRODUCTION}

The macaque monkey is an important model for cognitive and sensory neuroscience. During the last decade, investigative techniques, such as functional (fMRI), diffusion-weighted (DWI) and anatomical MRI have proven to be highly valuable for the study of functional processing and anatomy in these monkeys (1-12). However, several key differences exist between macaque and human imaging studies (13). Anesthesia is regularly used during scans (including functional scans) to mitigate body motion, which otherwise causes significant susceptibility artifacts and temporal fluctuations in the MR signal. Furthermore, the smaller head size of the macaque requires smaller voxel sizes to image with the same anatomical detail. Finally, the distance of the receive coils to the brain is large compared with that in humans because of the large temporal muscles of macaque monkeys and the head fixation post. Consequently, the signal-to-noise ratio (SNR) per voxel is inherently lower for macaque MRI.

An increase in SNR has been achieved for macaque MRI using field strengths of 4.7 and $7 \mathrm{~T}(5,13,14)$, thus requiring an expensive high-field scanner. Instead of increasing the field strength of the scanner, coil receiver array technology has been shown to be a valuable and cost-effective way to improve sensitivity and even encoding capabilities in MRI (15-18). Such multi-channel coils (with four to eight receive channels) have recently been introduced for functional and diffusion-weighted macaque imaging (19-30). Simulations for human applications have shown that the theoretical maximum SNR in the center of a spherical phantom is closely approached at $3 \mathrm{~T}$ when approximately 12 array elements are
* Correspondence to: W. Vanduffel, Department of Radiology, Harvard Medical School, Massachusetts General Hospital, Building 149, Room 2301, 13th Street, Charlestown, MA 02129, USA.

E-mail:wim@nmr.mgh.harvard.edu

a T. Janssens, B. Keil, P. Serano, A. Mareyam, J. A. McNab, L. L. Wald, W. Vanduffel

A.A. Martinos Center for Biomedical Imaging, Department of Radiology, Massachusetts General Hospital, Charlestown, MA, USA

b T. Janssens, W. Vanduffel

Laboratory for Neuro- and Psychophysiology, K.U. Leuven Medical School, Campus Gasthuisberg, Leuven, Belgium

c B. Keil, J. A. McNab, L. L. Wald, W. Vanduffel

Harvard Medical School, Boston, MA, USA

d J. A. McNab

R.M. Lucas Center for Imaging, Department of Radiology, Stanford University, Stanford, CA, USA

e L. L. Wald

Harvard-MIT Division of Health Sciences and Technology, Massachusetts Institute of Technology, Cambridge, MA, USA

+ These authors contributed equally to this work.

Abbreviations used: $A B S$, acrylonitrile butadiene styrene; $B W$, bandwidth; cov-rSoS, noise-covariance-weighted root sum-of-squares; DWI, diffusionweighted imaging; fMRl, functional MRl; FOV, field of view; G, geometry factor; Gd-DTPA, gadolinium diethylenetriaminepentaacetate; MPRAGE, magnetization prepared rapid gradient echo; $N_{s b}$ number of slices; $Q$, quality factor; $R$, acceleration rate; $R F$, radiofrequency; $R O I$, region of interest; SENSE, sensitivity encoding; SNR, signal-to-noise ratio; $T I$, inversion time; tSNR, temporal SNR. 
used (31). Many more elements are required to approach the maximum SNR in the periphery. Considering that the array coils commonly used to image the monkey brain consist of four to eight channels, a larger number of coil elements could potentially increase the SNR in both peripheral and central brain regions. In addition, the relatively low number of channels commonly employed, compared with the latest developments in RF technology for human MRI (64- and 96-channel coils) $(32,33)$, limit the potential for highly accelerated imaging in monkey MRI.

Multi-channel array coils for macaque brain imaging typically consist of a 'clam-shell' geometry with two curved formers housing array elements positioned on each side of the head $(27,34)$. The goal of this type of design is to better fit individual monkeys, whose head size/shape varies more significantly across individuals and adult ages than the human head because of the degree of musculature. Although the 'clam-shell' design provides high local SNR in the brain periphery, the intrinsic gap between the two coil shells results in local SNR decreases, especially in the frontal and occipital regions.

Long anesthesia sessions can be tedious and can require intubation of the monkey. As a result of considerations concerning both the well-being of the animals and the economic costs of scanning, we prefer to keep the anesthesia as short as possible, but also wish to retain the same SNR. To help to achieve this goal, we designed, constructed and evaluated a 22-channel receive array with a built-in local Helmholtz transmit coil for MRI of anesthetized macaques. The coil set-up was designed for the use in state-of-the-art clinical 3-T scanners with or without a strong human head-sized gradient insert. The receive elements of the 22-channel coil covered most of the monkey's head, including the eyes, the back of the head and the area in which a possible head post would probably be placed. The feasibility of high-resolution imaging in a highly accelerated acquisition scheme was evaluated in in vivo monkey brain measurements for SNR, G factor and noise correlation. Furthermore, the coil array's ability to obtain functional and multi-parametric imaging data (e.g. DWI) was investigated. We demonstrate that the use of accelerated data acquisition via the 22-channel phased array allows for shorter scan sessions, whilst still obtaining highresolution MR data.

\section{METHODS}

\section{Animal model}

The subject for this study was a male rhesus monkey (Macaca mulatta; $10.5 \mathrm{~kg}$ ). All procedures were approved by the Massachusetts General Hospital Subcommittee on Research Animal Care (protocol 2003N000338; P. I. Vanduffel) and are in accordance with the National Institutes of Health Guidelines for the Care and Use of Laboratory Animals. Anesthesia was induced and maintained with ketamine (Ketalar ${ }^{\circledR}$; Pfizer, New York, NY, USA) supplemented with dexmedetomidine (Dexdomitor ${ }^{\circledR}$; Pfizer). During the sessions, the monkey's body was heated using a heating pad. The monkey's heart rate, respiration and blood pressure were continuously monitored during the scans.

\section{2-channel receive coil}

The coil construction is analogous to that of previously reported human arrays $(33,35)$. The array was placed on a helmet printed from acrylonitrile butadiene styrene (ABS) using a rapid-prototyping three-dimensional printer (Dimension SST 1200es; Dimension, Inc., Eden Prairie, MN, USA). The close-fitting helmet was created by expanding a subsampled model of a monkey's head by $5 \mathrm{~mm}$. The subsampled head model (Fig. 1a) was created based on an MR image acquired with an ultra-short TE pulse sequence (TR/TE1/TE2/flip angle $\alpha=6.1 \mathrm{~ms} / 0.1 \mathrm{~ms} / 3.8 \mathrm{~ms} / 14^{\circ}$; isotropic voxel size, $0.8 \mathrm{~mm}$ ) to optimize the contrast at the air-skin boundary. For this scan, we used one of the larger monkeys in our laboratory $(11 \mathrm{~kg})$ to ensure that monkeys of all sizes would fit into the helmet. A surface model (in stl-format) was created from the image using Slicer 3 software (http://www.slicer.org) (36). Afterwards, the high-resolution surface was subsampled to reduce the complexity of the model. The head model was also printed and filled with physiological saline and $0.5 \mathrm{~mol} / \mathrm{L}$ gadolinium diethylenetriaminepentaacetate (Gd-DTPA) (MagnisvistVC; Bayer Schering Pharma, Berlin, Germany) adjusted to $T_{1} \sim 300 \mathrm{~ms}$. This monkey head phantom was used during coil bench tests and initial scanning tests. The helmet itself was designed to constrain the monkey in the sphinx position inside the scanner (1). This position is well-suited for anesthetized experiments as it stretches a

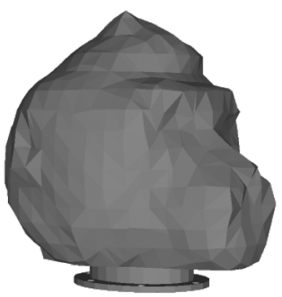

subsampled head phantom

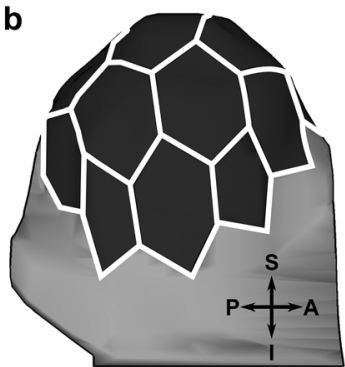

coil geometry, side view

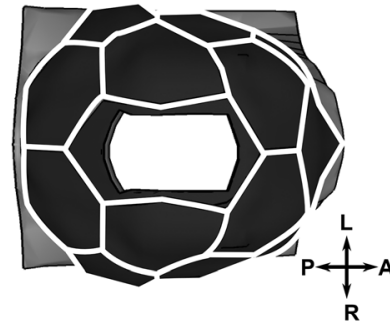

coil geometry, top view

Figure 1. Design of the 22-channel array coil for macaque monkeys. (a) The subsampled head model, obtained from an MR image, which was used to design the helmet. The subsampling was required to reduce the complexity for the model. In addition, this head model was three dimensionally printed and filled with physiological saline and $0.5 \mathrm{~mol} / \mathrm{L} \mathrm{Gd-DTPA} \mathrm{(MagnisvistVC;} \mathrm{Bayer} \mathrm{Schering} \mathrm{Pharma,} \mathrm{Berlin,} \mathrm{Germany)} \mathrm{adjusted} \mathrm{to} T_{1} \sim 300$ ms for coil testing. (b) The side and top view of the geometric tiling pattern of the 22 elements containing four pentagons, 17 hexagons and one strongly stretched hexagon (around the opening for the head post). These polygons were used as landmarks for the placement of the near-circular coil elements. The radius of the elements stretched out $\sim 0.5 \mathrm{~cm}$ further than the polygons to achieve the optimal overlap between nearest-neighbor elements. It should be noted that these images only show the part of the helmet that is covered by coil elements. A, anterior; I, inferior; L, left; $\mathrm{P}$, posterior; R, right; $\mathrm{S}$, superior. 
the neck of the monkey and intubation is not required for short scan sessions, although the helmet was designed to be compatible with the placement of an intubation tube for longer scan sessions. An opening was created on the top of the coil to allow for a possible head post (see Fig. 2a). In addition, the back of the helmet was designed to fit around the shoulders of the monkey in the sphinx position and to maximize the space available for electronics within the helmet. The front of the helmet contained an opening to allow free access to the mouth and for a possible intubation tube. The outside of the helmet was encased in ABS plastic to cover the electronics and to mount the coil in the scanner (see Fig. 2b). The coil fitted in a rectangular tray to ensure efficient coil mounting in a clinical MRI scanner, with or without a human gradient insert.

The position of the coil elements on the helmet was determined using a tiling pattern that was printed onto the helmet. This pattern consisted of four pentagons, 17 hexagons and one strongly stretched hexagon (around the large opening for the head post) (see Fig. 1b). The sizes of the tiles were adapted to completely cover the dorsal and lateral aspects of the monkey's head. The elements were formed manually from 16-AWG tinplated copper wires. The radius of all near-circular elements extended $\sim 0.5 \mathrm{~mm}$ further than the corresponding polygon to ensure the critical overlap for geometric decoupling. At the locations at which the loops crossed over, arches were bent into the wire to prevent them from touching. The loops contained two gaps located opposite one another for placement of the electronic components, which were positioned on small printed circuit boards that were manufactured with a rapid prototyping circuit board router (T-Tech-7000; T-Tech, Inc., Norcross, GA, USA). One circuit board contained the tuning capacitor $\mathrm{C}_{1}$ (BFC5 808 11339; Vishay Intertechnology, Inc., Malvern, PA, USA), whereas, on the other, the matching and detuning circuit was constructed. The latter consists of two identical capacitors $\left(C_{2}\right.$ and $\mathrm{C}_{3}$; Series 11; Voltronics, Danville, NJ, USA), a PIN diode (MA4P4002B-402; Macom, Lowell, MA, USA) and a hand-wound inductor. A short semi-rigid coaxial cable was used to connect the output circuit to the preamplifier (Siemens AG, Healthcare Sector, Erlangen, Germany). The optimal preamplifier decoupling was ensured by carefully controlling the length of the coaxial cable $(\sim 42 \mathrm{~mm})$. This electronic coil circuit has previously been described in detail $(35,37)$.

\section{Helmholtz transmit coil}

A local transmit coil is often used for monkey MRI, rather than the scanner's body transmit coil. This sort of local coil reduces the cable currents on otherwise long cables. It has the additional advantage that it can be used with a stronger human head gradient insert. When the insert gradient coil is used, the body
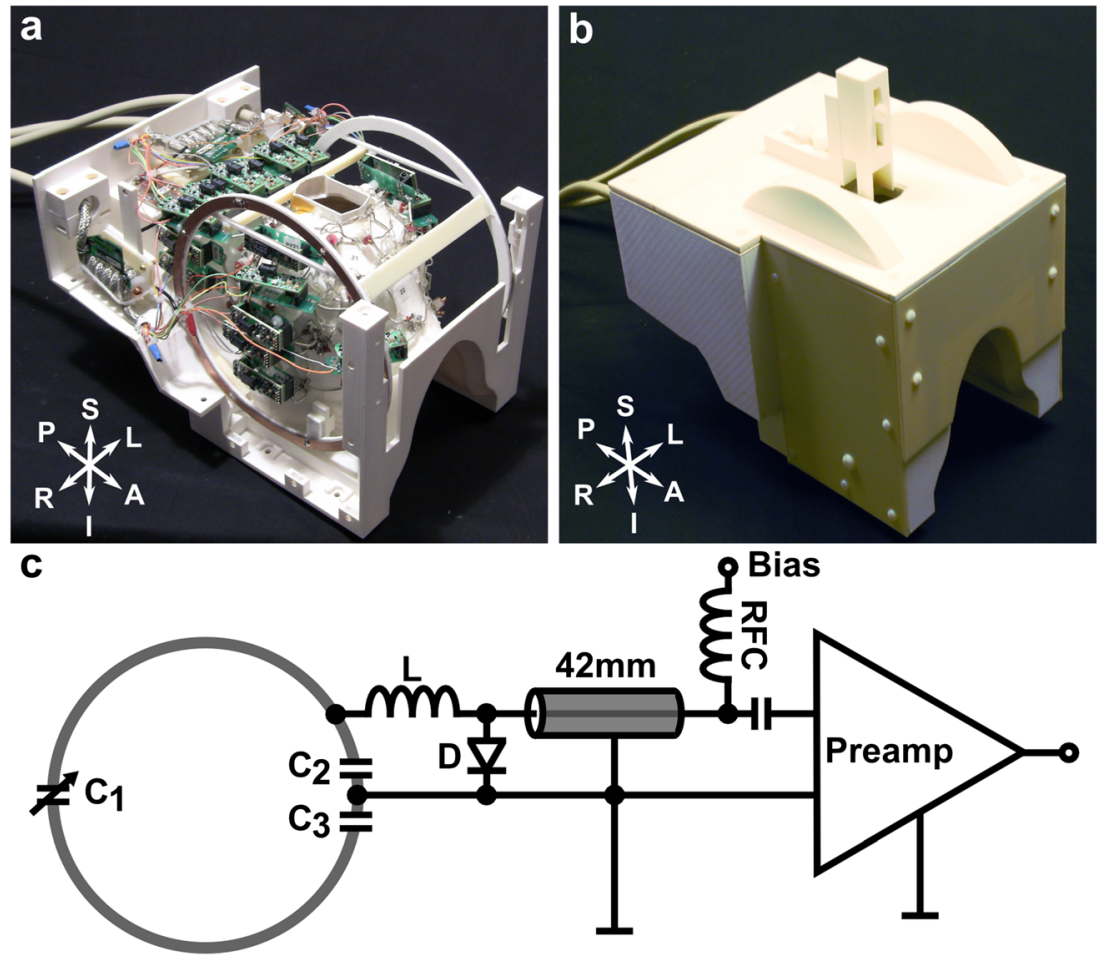

Figure 2. The 22-channel phased-array coil for macaque monkeys. (a) The inside of the array coil. All elements are arranged and secured in an orderly manner to avoid interactions between the elements and the effects of vibration in the scanner. All elements were packed as closely as possible to minimize the size of the coil. The Helmholtz transmit loops are visible on the lateral aspect of the coil. (b) The 22-channel coil completed with the external housing. The opening in front provides space for intubation equipment. On the top of the coil, the head post fixation system is visible. The position of the fixation system can be adjusted in the A-P and S-I directions to optimally position the heads of different monkeys inside the coil. The posterior part of the coil is shaped to fit the shoulders of the monkey comfortably. The helmet was designed to fit into a human head gradient insert system without touching the bore. (c) Schematic circuit of an individual channel. The coil element uses three capacitors: $C_{1}$ represents the variable tuning capacitor; impedance matching is provided by capacitor $C_{2}$ and capacitor $C_{3}$. A detuning trap is formed around $C_{2}$ using a handwound inductor $L$ and a diode $D$. The length of the coaxial cable ( $42 \mathrm{~mm}$ ) was empirically determined to ensure an optimal impedance transformation for preamp decoupling. A, anterior; I, inferior; L, left; $P$, posterior; R, right; RFC, radiofrequency choke; S, superior. 
transmit coil is disabled by default. In the current study, we constructed a Helmholtz transmit coil to create a more homogeneous $B_{1}$ excitation field compared with that of the single transmit loop commonly used in monkey MRI $(20,25,26)$. In addition, the design of the Helmholtz pair leaves more space near the monkey's head for the receive coil relative to a horizontally placed single loop transmit coil (see Fig. 2).

The transmit coil, shown schematically in Figure 3a, consists of two loops of $200 \mathrm{~mm}$ in size (outer diameter), which are mounted in the sagittal plane on either side of the helmet, $140 \mathrm{~mm}$ from each other (see Fig. 2a). Although we attempted to keep the distance between the loops as close as possible to the Helmholtz condition (half of the diameter of the loops) whilst fitting the head of the monkey, the actual inter-loop distance was slightly larger. Each loop was made of FR4 circuit board material; the circular path had a width of $10 \mathrm{~mm}$ and comprised eight symmetrically distributed tuning capacitors. Specifically, seven fixed capacitors of $33 \mathrm{pF}$ were used and one variable capacitor of 1-15 pF was added for exact tuning to $123.25 \mathrm{MHz}$. A shared matching circuit placed in the middle between the two loops provided matching to $50 \Omega$. Prior to construction of the transmit coil, we compared the $B_{1}^{+}$field homogeneity between the Helmholtz pair as designed and a horizontal single-loop transmit coil using a full-wave computational approach (HFSS v13; Ansoft, Canonsburg, PA, USA). The geometrical topologies of the simulated coils, including the size and position of the capacitors, were carefully modeled.

\section{One-channel and four-channel coils}

To quantify the benefits of the increased number of loop elements, the performance of the constructed 22-channel coil was compared with that of both a horizontal receive-only single-channel coil $(d=10 \mathrm{~cm})$ and a tight-fitting four-channel $(d=6 \mathrm{~cm})$ array coil. The single-loop coil was constructed from printed circuit board and contained 12 equally spaced capacitors. This single-loop coil was used in combination with the body transmit (without head gradient present). The four-channel coil consisted of two panels, each with two channels on each side of the monkey's head (34), not covering the eyes or the top and back of the head. The design of the electronics in the loops of the four-channel coil was analogous to that of the elements in the 22-channel coil. The fourchannel coil was used in combination with a single-loop horizontal detunable transmit coil (inner diameter $d=15 \mathrm{~cm}$; thickness, $1 \mathrm{~cm}$ ).

\section{Coil bench tests}

The procedures for bench testing during the construction of massively parallel detector arrays have been reviewed in detail recently $(37,38)$. Active detuning was first optimized for all the elements by measuring S21 between two decoupled ( 80 dB) inductive probes slightly coupled to the array element under test. Second, the overlap of nearest-neighbor loops was empirically adjusted to optimize geometric decoupling by a direct S21 measurement between pairs of elements with all other elements of the array detuned. In total, we performed three iterations of the S21 measurements to ensure the critical overlap between neighboring channels. Geometric decoupling can minimize the mutual reactance between a maximum of three neighboring elements (17). Therefore, the method of preamplifier decoupling was used to reduce the interaction between the (non-overlapping) channels. We measured the preamplifier decoupling of a given loop with all other loops detuned (26). Finally, the ratio of unloaded-to-loaded quality factors $\left(Q_{U} / Q_{L}\right)$ was measured for an average coil element size $(6 \mathrm{~cm})$. The $Q$ factors were measured with a dual-loop probe that lightly coupled into the loop under investigation (33), with no coaxial cable or preamplifier present. The $Q_{U} / Q_{L}$ ratios were tested on an isolated loop (no other elements present) and on a loop within the populated, but detuned, array.

\section{MR data acquisition and analysis}

Anesthetized monkey data were acquired with the 22-channel coil on a 3-T clinical MRI scanner (MAGNETOM, Trio - a Tim system, Siemens AG). We also acquired data from the same monkey using both the single-channel horizontal receive-only coil and the four-channel array coil. The SNR, noise correlation and $G$ factor were measured using proton density-weighted gradient echo images $\left[\mathrm{TR} / \mathrm{TE} / \alpha=30 \mathrm{~ms} / 6 \mathrm{~ms} / 30^{\circ}\right.$; slice, $1.5 \mathrm{~mm}$; 40 slices; matrix, $128 \times 128$; field of view (FOV), $128 \times 128 \mathrm{~mm}^{2}$; bandwidth (BW), $200 \mathrm{~Hz} /$ pixel]. Noise covariance information was acquired using the same pulse sequence, but with no RF excitation. SNR was calculated for images formed by combining the noise-covariance-weighted root sum-of-squares (cov-rSoS) of the individual channel images $(15,39)$ in Matlab (The Mathworks, Inc., Natick, MA, USA). The G-factor maps were used to assess noise amplification in sensitivity encoding (SENSE) reconstructions of the accelerated images. They were estimated from the complexvalued coil sensitivity profiles and the coil channel noise covariance matrix (16). The image FOV was chosen to tightly fit
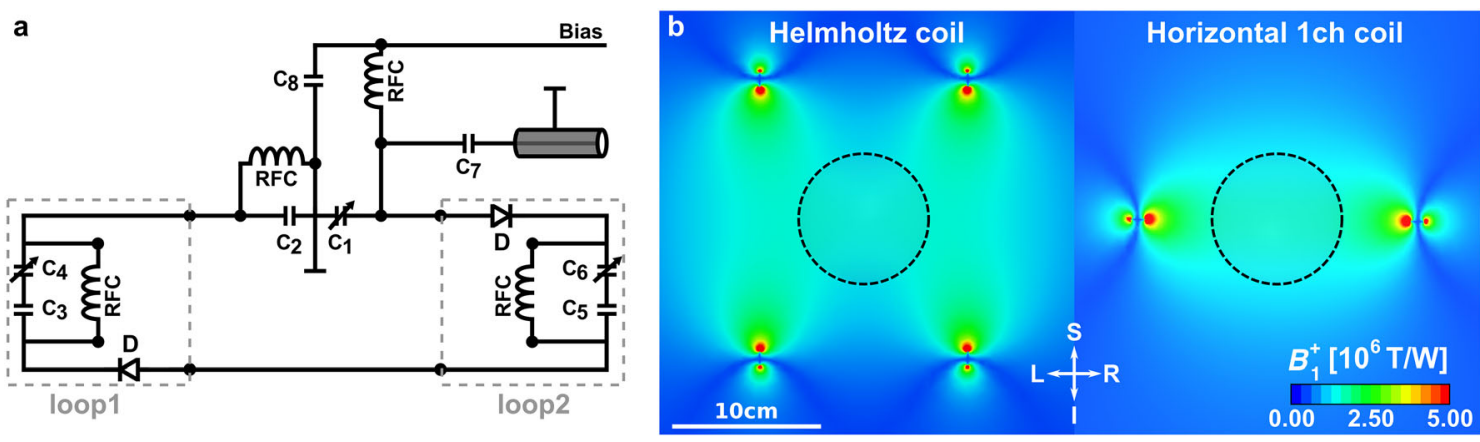

Figure 3. The Helmholtz transmit coil. (a) Schematic circuit of the Helmholtz transmit coil. $C_{1}$ and $C_{2}$ provide impedance matching, $C_{3-6}$ tune the loops. During the transmit phase, the two diodes $D$ are switched to activate the transmit coil, whereas the four radiofrequency chokes (RFC) ensure the closed path for the direct current. (b) Simulation of the $B_{1}^{+}$field generated by the Helmholtz coil and the horizontal single-loop transmit coil. The broken line indicates the position of a spherical region of interest of $9 \mathrm{~cm}$ in diameter in the center. 
the head $\left(96 \times 96 \mathrm{~mm}^{2}\right)$ to avoid excessive image encoding and to maximize aliasing in the accelerated image acquisition characterized by the $G$-factor map. A $5 \times 5$ pixel median filter was applied to the map to avoid the bias from outliers.

Next, we examined the performance of the 22-channel array for fast and high-resolution fMRI, DWI and anatomical imaging. Gradient recalled echo-echo planar images were acquired with a human head gradient insert (AC88; $80 \mathrm{mT} / \mathrm{m}$; slew rate, $400 \mathrm{~T} / \mathrm{m} / \mathrm{s}$; Siemens AG) at an isotropic resolution of $1 \mathrm{~mm}$ for acceleration rates $R=1-4$. Parameter values appropriate for functional imaging using blood oxygenation level-dependent contrast were chosen: axial slices; TR/TE $/ \alpha=3 \mathrm{~s} / 30 \mathrm{~ms} / 75^{\circ}$; matrix, $104 \times 104$; BW, $1924 \mathrm{~Hz} /$ pixel; 100 time points; partial Fourier = 7/8; number of slices $\left(N_{\mathrm{s}}\right)$ was maximized for each acceleration rate $R\left(R=1, N_{\mathrm{sl}}=31\right.$; $\left.R=2, N_{\mathrm{sl}}=41 ; R=3, N_{\mathrm{sl}}=46 ; R=4, N_{\mathrm{sl}}=49\right)$. The images were reconstructed online using GRAPPA (40). To quantify the temporal stability of the gradient recalled echo-echo planar images, the temporal SNR (tSNR) was determined for each voxel as the mean value across the 100 time points divided by that voxel's temporal standard deviation using Matlab.

In addition, high-resolution diffusion tensor images were acquired over $30 \mathrm{~min}$, again using the head gradient insert. Singleshot, twice-refocused diffusion-weighted spin-echo echo planar two-dimensional images were acquired at $0.7 \times 0.7 \times 1.5 \mathrm{~mm}^{3}$ using TR/TE $=3000 / 86 \mathrm{~ms}$, matrix size of $168 \times 1130, R=2,21$ slices, $\mathrm{BW}=960 \mathrm{~Hz} /$ pixel, two averages of 256 directions at $b=1000 \mathrm{~s} / \mathrm{mm}^{2}$ and $50 b=0$ images interspersed every nine volumes. Slices were again oriented axially with the monkey in the sphinx position, and the images were reconstructed online using GRAPPA.

Finally, high-resolution, accelerated, $T_{1}$-weighted, threedimensional, magnetization prepared rapid gradient echo (MPRAGE) images [TR/inversion time $(\mathrm{TI}) / \mathrm{TE} / \alpha=2.7 \mathrm{~s} / 850 \mathrm{~ms} / 3.74 \mathrm{~ms} / 9^{\circ}$; matrix, $512 \times 512 \times 240$; isotropic voxel size, $400 \mu \mathrm{m}$; BW = $180 \mathrm{~Hz} /$ pixel; $R=3$; online reconstruction using GRAPPA; total acquisition time, 7 min $14 \mathrm{~s} ; 12$ averages] were obtained and segmented using standard FreeSurfer 5.0 software tools (41). For the sake of comparison, a single MPRAGE image with identical scan parameters, except for $R=1$, was also acquired (acquisition time, $14 \min 26 \mathrm{~s}$ ).

\section{RESULTS}

The results of the Helmholtz coil simulation are plotted in Figure $3 \mathrm{~b}$. Within a range of $90 \mathrm{~mm}$ from the center, the standard deviations, normalized by the mean of the simulated $B_{1}^{+}$field along the right-left axis, were $1.6 \%$ and $2.7 \%$ for the Helmholtz and the single loop coil, respectively. Along the superior-inferior axis, these values were $7.3 \%$ and $15 \%$, respectively.

The receive-coil bench testing showed geometric decoupling between nearest-neighbor elements in the range of -13 to $-17 \mathrm{~dB}$, with an average of $-15 \mathrm{~dB}$. Preamplifier decoupling added an additional approximately $-20 \mathrm{~dB}$ of decoupling between all channels, on average. Active detuning during the excitation pulse was measured at $>40 \mathrm{~dB}$ on the bench. The $Q$-factor measurement resulted in $Q_{U} / Q_{L}=256 / 62=4.1$ for an isolated element, whereas, for an element with six neighbors, $Q_{U} / Q_{L}=235 / 62=3.8$, indicating sample noise dominance.

The element decoupling was verified in vivo by measuring the noise correlation coefficient matrix for the 22-channel coil (Fig. 4). The average noise correlation coefficient was $22 \%$. In general, the highest values observed in the noise correlation matrix originated from nearest-neighbor elements.

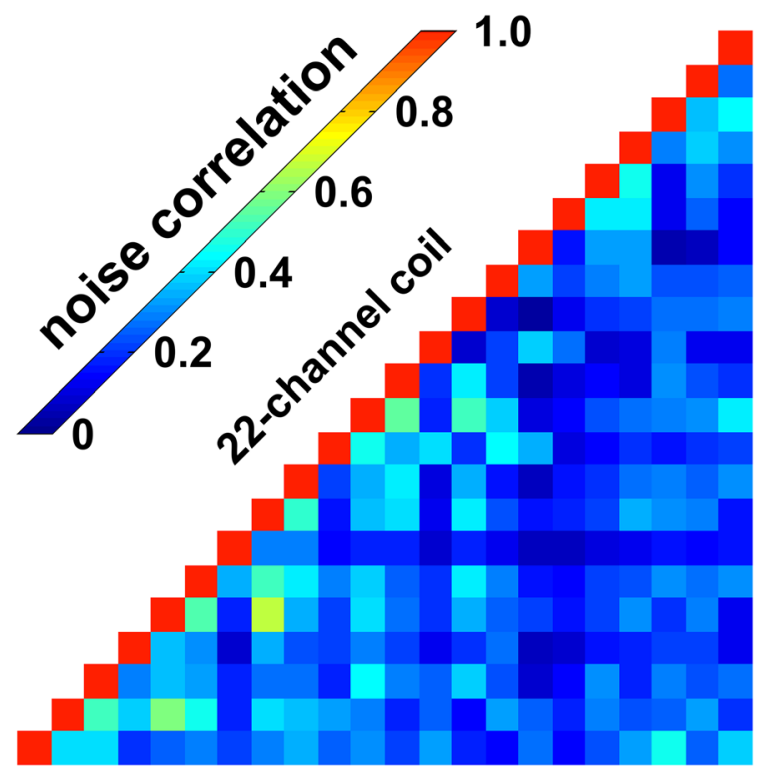

Figure 4. Noise correlation matrices of the 22-channel array. The noise correlation between any two individual channels averaged $22 \%$. Noise covariance information was acquired using the same pulse sequence as for the signal-to-noise ratio (SNR) measurement, but with no radiofrequency excitation.

In Figure 5, the in vivo SNR obtained from the 22-channel coil is compared with that from the single- and four-channel coils. The SNR was calculated as cov-rSoS. In a region of interest (ROI) covering the whole brain, the SNR gain factors were 1.9 and 1.5 compared with the single and four-channel coils, respectively.

The SNR gain depends on the location in the brain. Therefore, we calculated the SNR gains in two separate ROls covering the periphery and the center of the brain (see Fig. 5). In the ROI covering the periphery of the brain, the unaccelerated SNR improvement obtained with the 22-channel array measured 1.7- and 2.4-fold when compared with the four- and singlechannel coil, respectively. As a result of the improved coverage of the coil, an increase in SNR was also obtained in the center of the brain. In a circular ROI encompassing the central region of the brain, the SNR was increased 1.5-fold compared with that of both the four- and single-channel coil.

In addition to increasing the SNR, the higher channel count was also beneficial for parallel imaging. In Figure 6 , the in vivo $1 / G$ factor is shown in an axial plane for one- and twodimensional acceleration for both the four- and 22-channel array coils. Figure 6 also shows the ROls used to analyze the $G$ factors of the central and peripheral regions. Compared to the fourchannel coil, the 22-channel array produces lower $G$ factors at all locations in the head and for all accelerations, providing roughly one additional unit of acceleration for a given noise amplification factor. The average $G$ factors at the center and periphery are shown for both coils in Table 1.

Figures 7, 8 and 9 assess the performance of the array for fMRI, DWI and anatomical imaging, respectively. In Figure 7, singleshot two-dimensional echo planar images (isotropic resolution, $1 \mathrm{~mm}$ ) are shown for acceleration rates $R=1-4$ and typical scan parameters for blood oxygenation level-dependent contrast (see Methods). Up to $R=2$ or 3 , image quality and tSNR at these high resolutions seem to be sufficient for functional experiments. Image degradation, originating from noise contributions related to the $G$ factor, becomes more pronounced at $R=4$. As expected, 

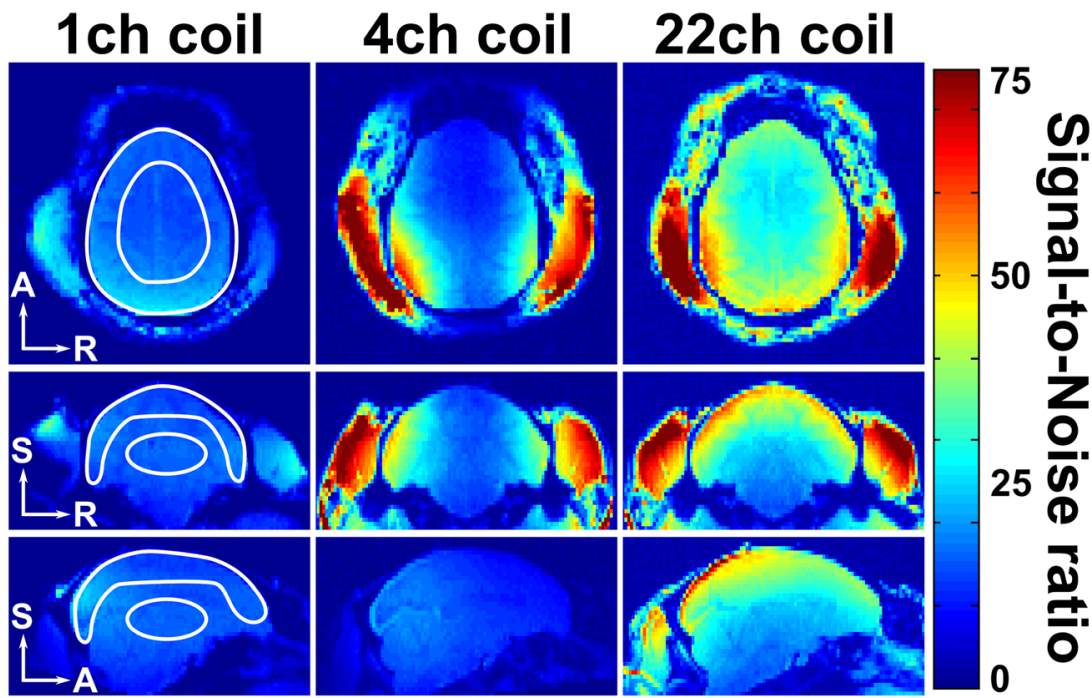

Figure 5. In vivo signal-to-noise ratio (SNR) comparisons in three orthogonal slices between the single-, four- and 22-channel coils. The SNR produced by the 22-channel coil is significantly higher than that of the other coils. The SNR maps were calculated for images formed from noise-covarianceweighted root sum-of-squares (cov-rSoS) of the individual channel images. The central and peripheral regions of interest are indicated on the images obtained with the single-channel coil. A, anterior; $R$, right; $S$, superior.
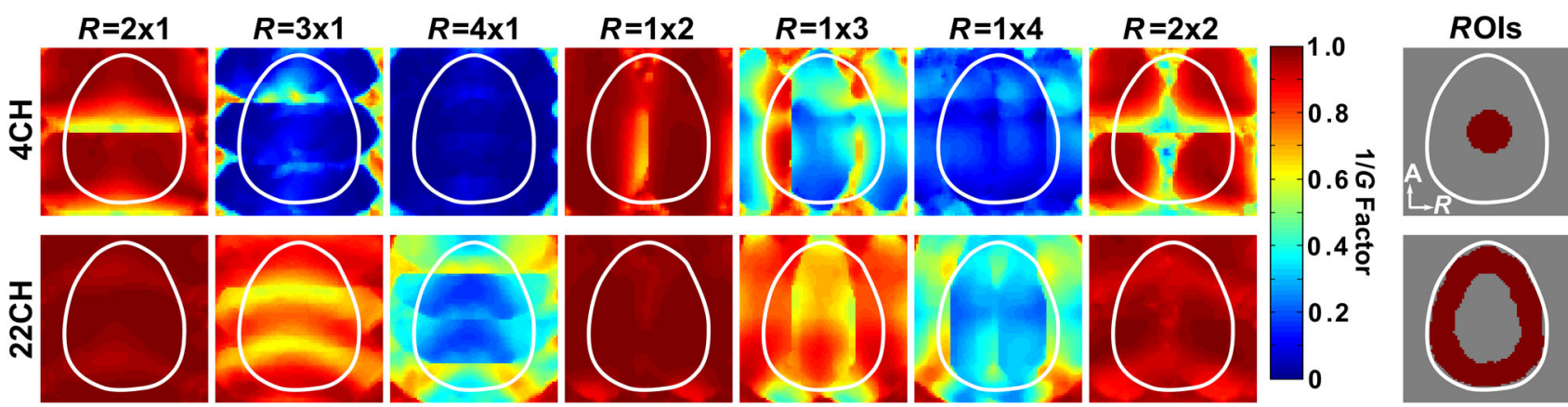

Figure 6. In vivo transverse maps of $1 / G$ factor obtained with the four- and 22-channel coils. The field of view was made as tight as possible around the monkey's head to avoid underestimation of the $G$ factors. The 22-channel array yields lower average and peak $G$-factor values relative to the fourchannel coil (see Table 1). Following the $G$-factor calculations, the results were masked with a region of interest (ROI) encompassing the entire brain. The central and peripheral ROls are indicated next to the $G$-factor images. A, anterior; $R$, right.

Table 1. Measured average $G$-factor values for the four- $(4-\mathrm{CH})$ and 22 -channel $(22-\mathrm{CH})$ arrays for one- (left-right or anteriorposterior direction) and two-dimensional acceleration directions in the periphery and center regions of interest (ROIs)

\begin{tabular}{|lcccccccc|} 
& & $\mathrm{R}=2 \times 1$ & $R=3 \times 1$ & $R=4 \times 1$ & $R=1 \times 2$ & $R=1 \times 3$ & $R=1 \times 4$ & $R=2 \times 2$ \\
\hline ROI = periphery & $4-\mathrm{CH}$ & 1.11 & 17.0 & 66.0 & 1.05 & 2.55 & 8.18 & 1.23 \\
& $2-\mathrm{CH}$ & 1.02 & 1.32 & 3.01 & 1.01 & 1.33 & 3.09 & 1.06 \\
$\mathrm{ROI}$ = center & $4-\mathrm{CH}$ & 1.32 & 11.0 & 35.6 & 1.16 & 3.40 & 8.10 & 1.94 \\
& $22-\mathrm{CH}$ & 1.01 & 1.35 & 4.68 & 1.02 & 1.43 & 3.46 & 1.04 \\
\hline
\end{tabular}

reduced image distortion from susceptibility-induced gradients is observed for higher $R$ factors. Furthermore, reduced temporal variance is expected in the time series as a result of image modulations from body and throat movements.

The gain in SNR obtained from the 22-channel coil can be used to compensate for the intrinsic drop in SNR during parallel image acquisition. This allows us to accelerate data acquisition by a factor of two or three, considerably reducing the scan time under anesthesia. The resulting MPRAGE images at an isotropic resolution of $0.4 \mathrm{~mm}$ are shown in Figure 8 . The quality of a single image obtained with the 22-channel array is still better than that of the horizontal single loop coil, even though it was collected in almost one-third of the scan time using $R=3$. At this acceleration rate, 12 scans with whole-brain coverage could be collected in $1.5 \mathrm{~h}$. The average image has sufficient SNR for segmentation in FreeSurfer with minimal manual input or 
a
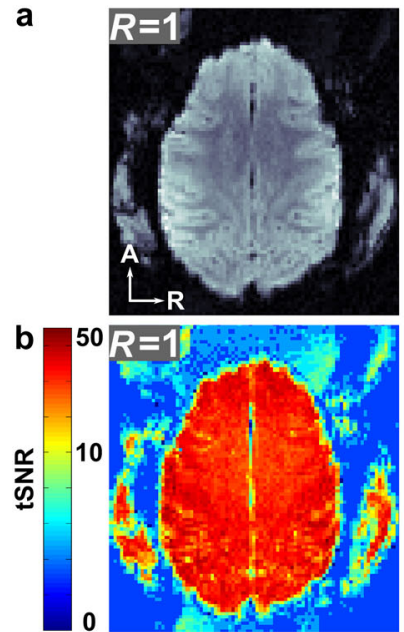
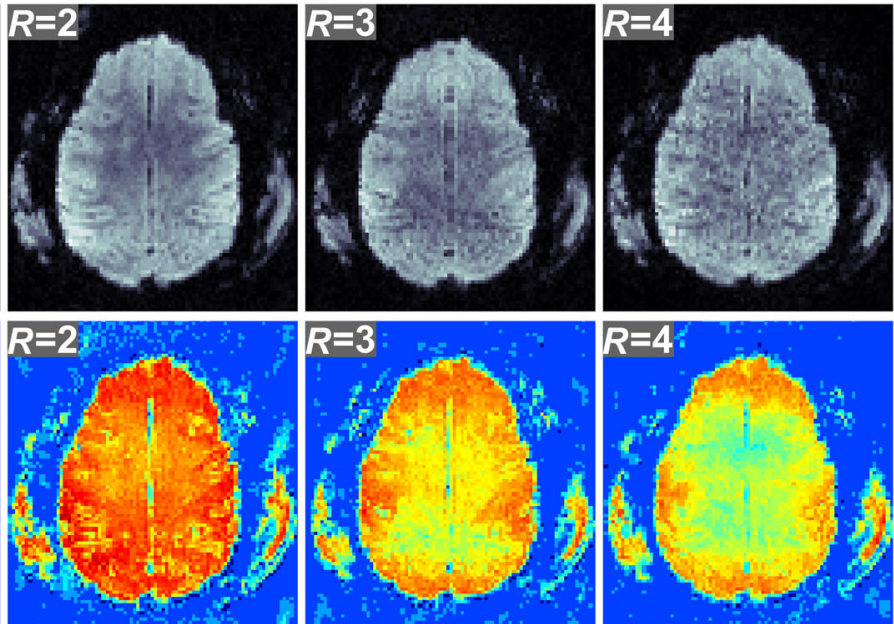
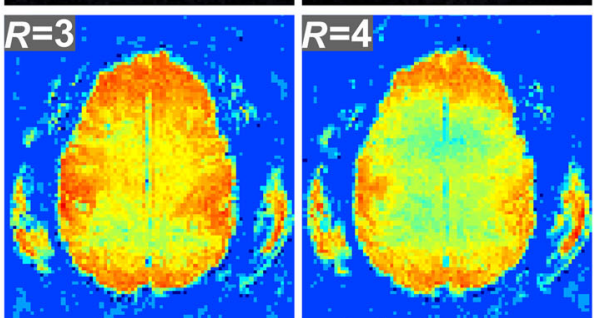

Figure 7. High-resolution (1 $\mathrm{mm}$ isotropic) functional images. (a) Single gradient recalled echo-echo planar imaging volumes acquired with the 22 -channel array at acceleration rates $R=1-4$ (acceleration direction, head-foot). As the acceleration rate is increased, the signal-to-noise ratio (SNR) decreases. The predicted noise enhancement pattern from the $G$-factor maps in Fig. 5 is shown. However, image distortions (stretching and blurring) in the front and back of the brain diminish with increasing acceleration. (b) Temporal SNR maps of the same slices and acceleration rates. $A$, anterior; $R$, right.
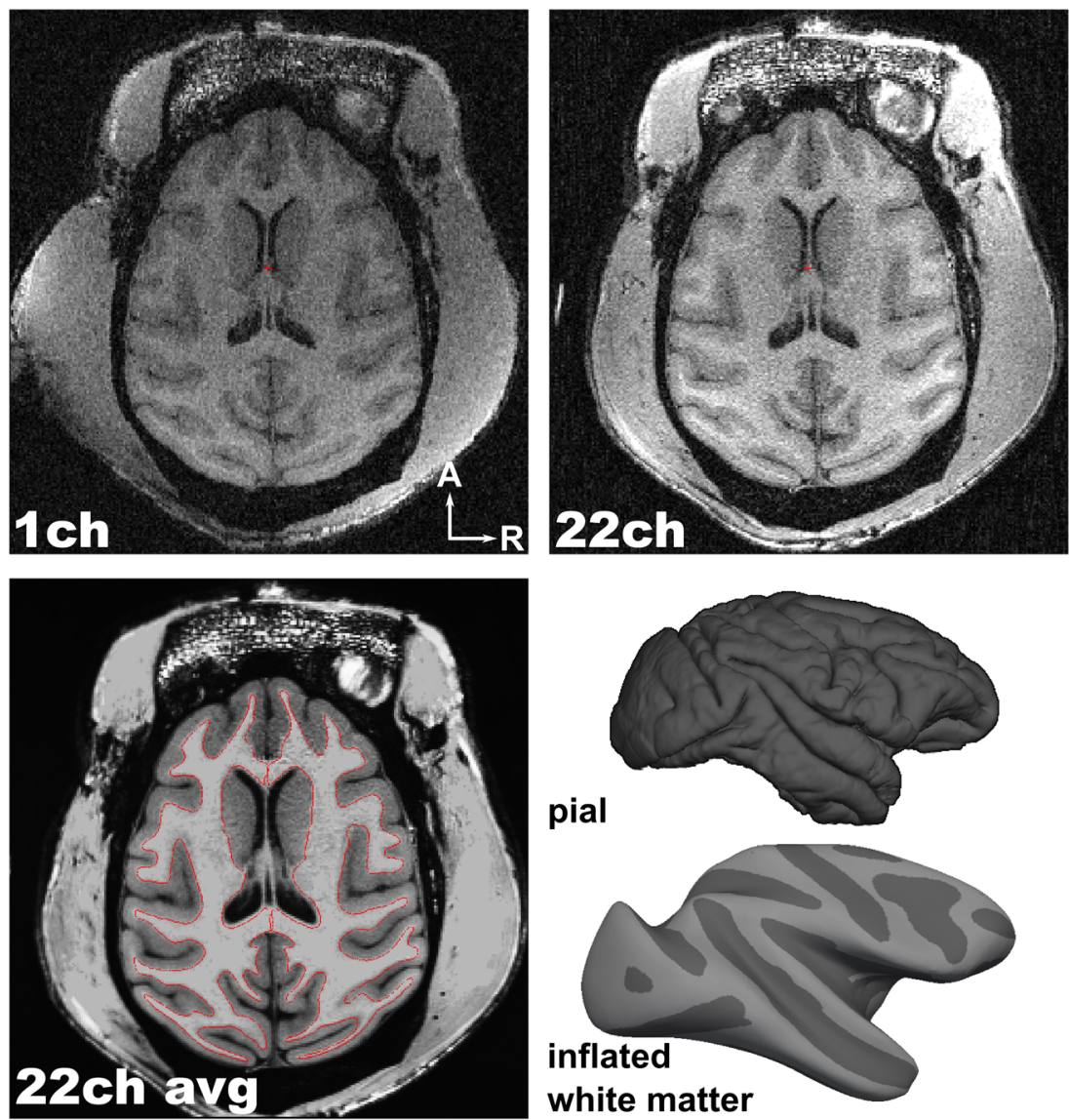

Figure 8. High-resolution ( $0.4 \mathrm{~mm}$ isotropic) anatomical magnetization prepared rapid gradient echo (MPRAGE) imaging. Top left: single volume obtained in 14 min with a horizontal single-channel coil. Top right: single volume acquired in 6 min with the 22-channel coil using $R=3$. Bottom left: average of 12 volumes obtained with the 22-channel array in less than $1.5 \mathrm{~h}$. The white matter segmentation by FreeSurfer is indicated in red. Bottom right: resulting surfaces derived from segmentation by FreeSurfer. Both the pial surface and the inflated white matter are shown. A, anterior; R, right.

corrections. The resulting segmentation shown in Figure 8 was achieved by adding only control points to the most anterior part of the orbitofrontal cortex between the eyes, and by limited manual editing to the white matter volume around the midbrain structures. This process required only $2-3 \mathrm{~h}$ of manual labor, which is relatively fast for the segmentation of monkey brains. 

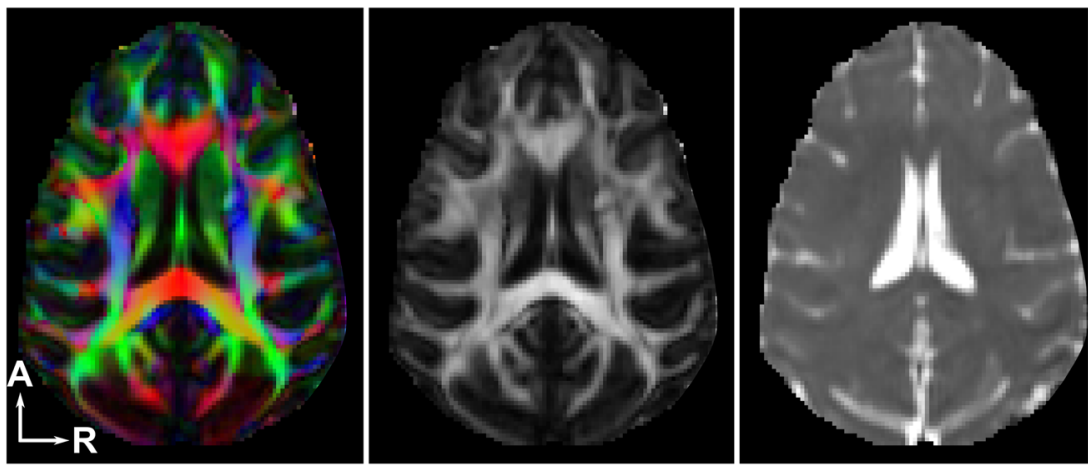

Figure 9. High-resolution $\left(0.7 \times 0.7 \times 1.5 \mathrm{~mm}^{3}\right)$ diffusion tensor images acquired with the 22-channel array. Diffusion tensor imaging including maps of the color-encoded direction (red, green and blue representing the L-R, A-P and S-I directions, respectively) (left), fractional anisotropy (middle) and mean diffusivity (right). Images were acquired at $3 \mathrm{~T}$ with a head insert gradient $\left(G_{\max }=80 \mathrm{mT} / \mathrm{m}\right)$. Imaging parameters were $R=2, b=1000 \mathrm{~s} / \mathrm{mm}^{2}, 256$ directions and two averages. The total scan time was 30-45 min. A, anterior; I, inferior; L, left; P, posterior; R, right; S, superior.

Finally, the performance of the 22-channel coil for DWI is illustrated in Figure 9. One of the horizontal slices of the color-coded direction, fractional anisotropy and apparent diffusion coefficient maps is shown. The image quality is sufficient to see detailed white matter structures throughout the whole brain. As a result of the higher SNR and acceleration rate $(R=2)$, this highresolution $\left(0.7 \times 0.7 \times 1.5 \mathrm{~mm}^{3}\right)$ diffusion dataset could be collected in less than $1 \mathrm{~h}$.

\section{DISCUSSION}

In this study, we have shown that similar highly parallel reception strategies to those used in humans $(15,18,33)$ can be achieved in the macaque. The 22-channel brain array was specifically designed for efficient imaging of macaque brain at $3 \mathrm{~T}$ (with or without a head gradient insert) with improved speed and spatial resolution for data acquisition. Furthermore, the value of the 22-channel coil with respect to various types of MR acquisitions ( $\mathrm{fMRl}$, anatomy and diffusion tensor imaging) was demonstrated.

The characterization of coil imaging properties included noise correlation, SNR and G-factor evaluation. The average in vivo noise correlation falls within the range of previously reported values for phased-array coils having large numbers of channels $(35,38)$.

The in vivo SNR obtained with the 22-channel array in the periphery of the brain was significantly higher than that obtained with a single loop coil (2.4-fold increase) or a four-channel array coil (1.7-fold increase). As a result of the increase in the number of channels and improved coverage around the top, front, back and lateral portions of the head, a 1.5-fold gain in SNR was found in a central ROI compared with either the single- or four-channel coil. An SNR increase in the center was expected because simulations have shown that the ultimate SNR (for a human-sized spherical geometry) in the center is only reached with 12 or more channels (31).

An identical four-channel coil to that described in the current study was used in previous studies $(26,34)$. In these studies, it was shown that the SNR increase using an eight-channel coil with a similar 'clam-shell' geometry did not drastically increase the SNR inside the brain compared with the four-channel coil because of the large temporal jaw muscles of macaque monkeys. However, when using the 22-channel array, a significant increase in SNR was observed because of the improved coverage around the head.
Other coil designs have been described recently for macaque fMRI, such as a flexible linear receive array (19) and implanted coils $(26,42)$. These coil designs are characterized by a very high local SNR because of the proximity of the coil to the brain, but often lack full brain coverage $(19,42)$, and result in an extremely nonhomogeneous SNR distribution in the images (26). These properties make these coil designs less suited for purposes such as tissue segmentation.

In this study, we compared the SNR performance of the 22channel coil with that of two commonly used coils in monkey MRI (the single- and four-channel coils). Another type of receive system that is often used for SNR comparison is a volume coil, e.g. a birdcage coil (38). In general, volume coils are characterized by a more homogeneous sensitivity profile throughout the brain, but with lower SNR compared with surface array coils $(18,19,38)$. By extrapolation of the results from other studies, we expect that the 22-channel coil also has a large increase in SNR in the outer periphery of the brain compared with a size-matched volume coil. However, in the center of the brain, we would expect only a slight gain in SNR (38). Nevertheless, the positioning of the monkey in the sphinx position and the commonly used head post system obstruct the design of a close-fitting birdcage volume coil.

In the final phase of coil characterization, the in vivo $G$ factor was measured. The $G$-factor maps show that acceleration rates of up to three can be achieved in both the anterior-posterior and left-right directions with the 22-channel coil. The improved $G$ factors over those of the four-channel coil are a consequence of the larger number of receive elements and improved coverage. This provides a stronger spatial modulation of the signal intensity, and thus an improved ability to unalias folded images (SENSE method) (16) or to synthesize spatial harmonics (SMASH or GRAPPA methods) (40).

An additional benefit of the 22-channel coil is that the helmet design, with integrated head post fixation, will greatly reduce the variability in coil positioning across scan sessions relative to previous monkey array coils with the 'clam-shell' geometry and a smaller number of elements.

In addition to the characterization of the performance of the 22-channel array, we have demonstrated its use in various common MR modalities: $\mathrm{FMRI}$, anatomical imaging and DWI. For all three modalities, high-resolution images were obtained with reduced scan times relative to single or four-channel coils. The 22-channel array has been shown to be especially suited 
for anatomical imaging and DWI. For example, in general, a highresolution (isotropic resolution of $0.4 \mathrm{~mm}^{3}$ ) anatomical MPRAGE image is used for the visualization of fMRI activations. Often a single loop coil is used for the acquisition of such an anatomical image because of the homogeneous SNR distribution, which facilitates the segmentation of gray and white matter regions. However, as shown in Figure 5, a single loop coil is limited with regard to SNR, so that a relatively large number (10-15) of high-resolution anatomical acquisitions (e.g. MPRAGE) are required. This necessitates a long scan session under anesthesia. By using the 22-channel array coil, the SNR is always higher and still sufficiently homogeneous for segmentation using FreeSurfer 5.0 (see Fig. 5).

The decreased scan time and increased SNR obtained when using the 22-channel receive coil are highly beneficial for various MRI studies employing anesthetized monkeys.

\section{Acknowledgements}

The authors thank H. Deng and R. Farivar for technical support during the scans. This work received support from Inter University Attraction Pole 6/29, Program Financing PFV/10/008, Geconcerteerde Onderzoeks Actie 10/19, Hercules funding, Fonds Wetenschappelijk Onderzoek (FWO)-Vlaanderen (G062208N10, G083111N10 and G043912N) and National Science Foundation (NSF) grant BCS-0745436. TJ is an aspirant of the FWO-Vlaanderen. The Martinos Center is supported by National Center for Research Resources grant P41RR14075.

\section{REFERENCES}

1. Vanduffel W, Fize D, Mandeville JB, Nelissen K, Van Hecke P, Rosen $B R$, Tootell RB, Orban GA. Visual motion processing investigated using contrast agent-enhanced $\mathrm{fMRI}$ in awake behaving monkeys. Neuron 2001; 32(4): 565-577.

2. Stefanacci L, Reber P, Costanza J, Wong E, Buxton R, Zola S, Squire L, Albright T. fMRI of monkey visual cortex. Neuron 1998; 20(6): 1051-1057.

3. Logothetis NK, Guggenberger H, Peled S, Pauls J. Functional imaging of the monkey brain. Nat. Neurosci. 1999; 2(6): 555-562.

4. Disbrow EA, Slutsky DA, Roberts TP, Krubitzer LA. Functional MRI at 1.5 tesla: a comparison of the blood oxygenation level-dependent signal and electrophysiology. Proc. Natl. Acad. Sci. USA 2000; 97(17): 9718-9723.

5. Logothetis NK, Pauls J, Augath $M$, Trinath $T$, Oeltermann A. Neurophysiological investigation of the basis of the fMRI signal. Nature 2001; 412(6843): 150-157.

6. Adluru N, Zhang H, Fox AS, Shelton SE, Ennis CM, Bartosic AM, Oler JA, Tromp do PM, Zakszewski E, Gee JC, Kalin NH, Alexander AL. A diffusion tensor brain template for rhesus macaques. Neuroimage 2012; 59(1): 306-318.

7. Makris N, Papadimitriou GM, van der Kouwe A, Kennedy DN, Hodge SM, Dale AM, Benner T, Wald LL, Wu O, Tuch DS, Caviness VS, Moore TL, Killiany RJ, Moss MB, Rosene DL. Frontal connections and cognitive changes in normal aging rhesus monkeys: a DTI study. Neurobiol. Aging 2007; 28(10): 1556-1567.

8. Moeller S, Freiwald WA, Tsao DY. Patches with links: a unified system for processing faces in the macaque temporal lobe. Science 2008; 320(5881): 1355-1359.

9. Arcaro MJ, Pinsk MA, Li X, Kastner S. Visuotopic organization of macaque posterior parietal cortex: a functional magnetic resonance imaging study. J. Neurosci. 2011; 31(6): 2064-2078.

10. Tsao DY, Freiwald WA, Knutsen TA, Mandeville JB, Tootell RB. Faces and objects in macaque cerebral cortex. Nat. Neurosci. 2003; 6(9): 989-995.

11. Bell AH, Hadj-Bouziane F, Frihauf JB, Tootell RB, Ungerleider LG. Object representations in the temporal cortex of monkeys and humans as revealed by functional magnetic resonance imaging. J. Neurophysiol. 2009; 101(2): 688-700.

12. Matsui T, Tamura K, Koyano KW, Takeuchi D, Adachi $Y$, Osada T, Miyashita Y. Direct comparison of spontaneous functional connectivity and effective connectivity measured by intracortical microstimulation: an FMRI study in macaque monkeys. Cereb. Cortex 2011; 21(10): 2348-2356.

13. Goense JB, Whittingstall K, Logothetis NK. Functional magnetic resonance imaging of awake behaving macaques. Methods 2010; 50(3): 178-188.

14. Pfeuffer J, Shmuel A, Keliris GA, Steudel T, Merkle H, Logothetis NK. Functional MR imaging in the awake monkey: effects of motion on dynamic off-resonance and processing strategies. Magn. Reson. Imaging 2007; 25(6): 869-882.

15. Roemer PB, Edelstein WA, Hayes CE, Souza SP, Mueller OM. The NMR phased array. Magn. Reson. Med. 1990; 16(2): 192-225.

16. Pruessmann KP, Weiger M, Scheidegger MB, Boesiger P. SENSE: sensitivity encoding for fast MRI. Magn. Reson. Med. 1999; 42(5): 952-962.

17. Wright SM, Wald LL. Theory and application of array coils in MR spectroscopy. NMR Biomed.. 1997; 10(8): 394-410.

18. Wiggins GC, Triantafyllou C, Potthast A, Reykowski A, Nittka M, Wald LL. 32-channel 3 Tesla receive-only phased-array head coil with soccer-ball element geometry. Magn. Reson. Med. 2006; 56(1): 216-223.

19. Goense J, Logothetis NK, Merkle H. Flexible, phase-matched, linear receive arrays for high-field MRI in monkeys. Magn. Reson. Imaging 2010; 28(8): 1183-1191.

20. Kolster H, Mandeville JB, Arsenault JT, Ekstrom LB, Wald LL, Vanduffel W. Visual field map clusters in macaque extrastriate visual cortex. J. Neurosci. 2009; 29(21): 7031-7039.

21. Ekstrom LB, Roelfsema PR, Arsenault JT, Kolster H, Vanduffel W. Modulation of the contrast response function by electrical microstimulation of the macaque frontal eye field. J. Neurosci. 2009; 29(34): 10 683-10 694.

22. Nelissen K, Borra E, Gerbella M, Rozzi S, Luppino G, Vanduffel W, Rizzolatti G, Orban GA. Action observation circuits in the macaque monkey cortex. J. Neurosci. 2011; 31(10): 3743-3756.

23. Nelissen K, Vanduffel W. Grasping-related functional magnetic resonance imaging brain responses in the macaque monkey. J. Neurosci. 2011; 31(22): 8220-8229.

24. Mantini D, Gerits A, Nelissen K, Durand JB, Joly O, Simone L, Sawamura H, Wardak C, Orban GA, Buckner RL, Vanduffel W. Default mode of brain function in monkeys. J. Neurosci. 2011; 31(36): 12 954-12 962.

25. Ekstrom LB, Roelfsema PR, Arsenault JT, Bonmassar G, Vanduffel W. Bottom-up dependent gating of frontal signals in early visual cortex. Science 2008; 321(5887): 414-417.

26. Janssens T, Keil B, Farivar R, McNab JA, Polimeni JR, Gerits A, Arsenault JT, Wald LL, Vanduffel W. An implanted 8-channel array coil for high-resolution macaque MRI at 3T. Neuroimage, 2012; 62(3): 1529-1536.

27. Janssens T, Vanduffel W, Kolster H. SNR optimization of MION fMRI in the anaesthetized monkey using an 8-channel PA-coil and accelerated imaging. Proceedings of the 17th Annual Meeting ISMRM, Honolulu, HI, USA, 2009; 1619.

28. Liu X, Zhu T, Gu T, Zhong J. Optimization of in vivo high-resolution DTI of non-human primates on a $3 T$ human scanner. Methods, 2010; 50(3): 205-213.

29. Khachaturian MH, Arsenault J, Ekstrom LB, Tuch DS, Vanduffel W. Focal reversible deactivation of cerebral metabolism affects water diffusion. Magn. Reson. Med. 2008; 60(5): 1178-1189.

30. Khachaturian MH. A 4-channel 3 Tesla phased array receive coil for awake rhesus monkey fMRI and diffusion MRI experiments. J. Biomed. Sci. Eng. 2010; 3(11): 1085-1092.

31. Wiesinger F, DeZanche N, Pruessmann KP. Approaching ultimate SNR with finite coil arrays. Proceedings of the 13th Annual Meeting ISMRM, Miami Beach, FL, USA, 2005; 672.

32. Wiggins GC, Polimeni JR, Potthast A, Schmitt M, Alagappan V, Wald LL. 96-Channel receive-only head coil for 3 Tesla: design optimization and evaluation. Magn. Reson. Med. 2009; 62(3): 754-762.

33. Keil B, Blau JN, Biber S, Hoecht P, Tountcheva V, Setsompop K, Triantafyllou C, Wald LL. A 64-channel 3T array coil for accelerated brain MRI. Magn. Reson. Med. 2012; doi:10.1002/mrm.24427.

34. Mareyam A, Blau J, Polimeni J, Keil B, Farivar R, Benner T, Vanduffel W, Wald LL. Eight-channel array coil optimized for functional imaging of awake monkeys at 7T. Proceedings of the 19th Annual Meeting ISMRM, Montreal, QC, Canada, 2011; 1823. 
35. Keil B, Alagappan V, Mareyam A, McNab JA, Fujimoto K, Tountcheva V, Triantafyllou C, Dilks DD, Kanwisher N, Lin W, Grant PE, Wald LL. Size-optimized 32-channel brain arrays for $3 \mathrm{~T}$ pediatric imaging Magn. Reson. Med. 2011; 66(6): 1777-1787.

36. Pieper S, Lorensen B, Schroeder W, Kikinis R. The NA-MIC Kit: ITK, VTK, Pipelines, Grids and 3D Slicer as an Open Platform for the Medical Image Computing Community. Proceedings of the 3rd IEEE International Symposium on Biomedical Imaging: From Nano to Macro, Arlington, VA, USA, 2006; 698-701.

37. Keil B, Wald LL. Massively parallel MRI detector arrays. J. Magn. Reson. 2013; 229: 75-89.

38. Keil B, Wiggins GC, Triantafyllou C, Wald LL, Meise FM, Schreiber LM, Klose KJ, Heverhagen JT. A 20-channel receive-only mouse array coil for a 3 T clinical MRI system. Magn. Reson. Med. 2011; 66(2): 584-595.

39. Kellman P, McVeigh ER. Image reconstruction in SNR units: a general method for SNR measurement. Magn. Reson. Med. 2005; 54(6): 1439-1447.

40. Griswold MA, Jakob PM, Heidemann RM, Nittka M, Jellus V, Wang J, Kiefer B, Haase A. Generalized autocalibrating partially parallel acquisitions (GRAPPA). Magn. Reson. Med. 2002; 47(6): 1202-1210.

41. Dale AM, Fischl B, Sereno MI. Cortical surface-based analysis. I. Segmentation and surface reconstruction. Neurolmage, 1999; 9(2): 179-194.

42. Logothetis $\mathrm{N}$, Merkle $\mathrm{H}$, Augath $\mathrm{M}$, Trinath $\mathrm{T}$, Ugurbil K. Ultra highresolution fMRI in monkeys with implanted RF coils. Neuron 2002; 35(2): 227-242. 